\title{
High Vaccine Coverage is Crucial for Preventing the Spread of Infectious Diseases During Mass Gathering
}

Susu M. Zughaier (szughaier@qu.edu.qa)

College of Medicine, Qatar University, Doha, Qatar PO Box 2713

\section{Background}

Vaccines are the most cost-effective intervention in public health as they prevent the spread of highly contagious infectious diseases (1). Because of vaccine implementation and high coverage, Measles was eradicated in 2000 (1), however the recent reappearance of measles in the United States, Europe and globally is alarming (2).

\section{Vaccine Coverage Reduces Burden of Disease}

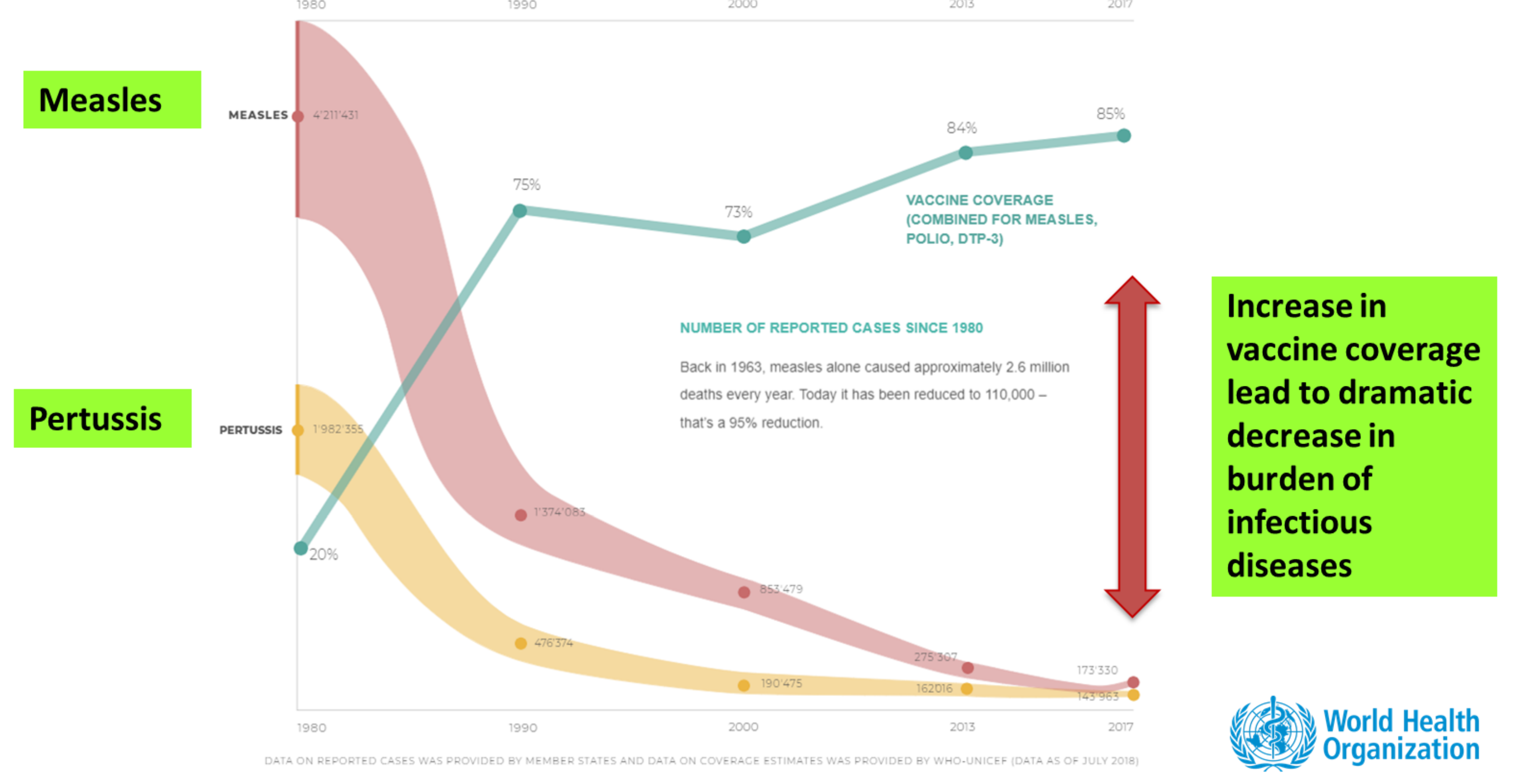

\section{Measles Resurgence due to Reduced Vaccine Coverage}

The resurgence of Measles,

Diphtheria and Mumps is due

to a reduction in vaccine coverage and herd immunity.

Vaccine hesitant parents, antivaxxers, and fake news on vaccines are driving the surge in those infectious diseases.

The World Health

Organization issued the Global Vaccine and Immunization Action Plan to reiterate the importance of vaccine implementation and coverage for several vaccinepreventable infectious diseases in the world.

Vaccine coverage induces community protection (Herd Immunity)

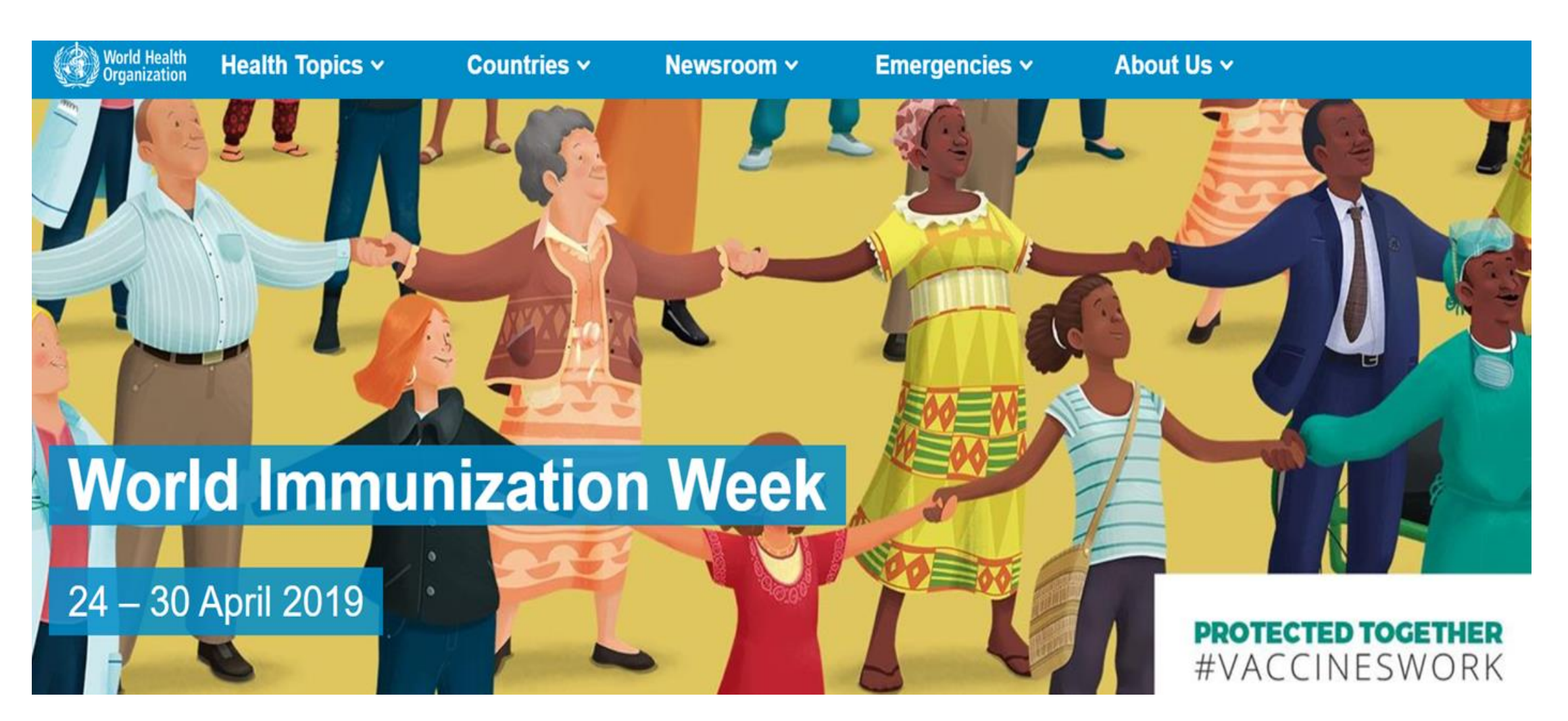

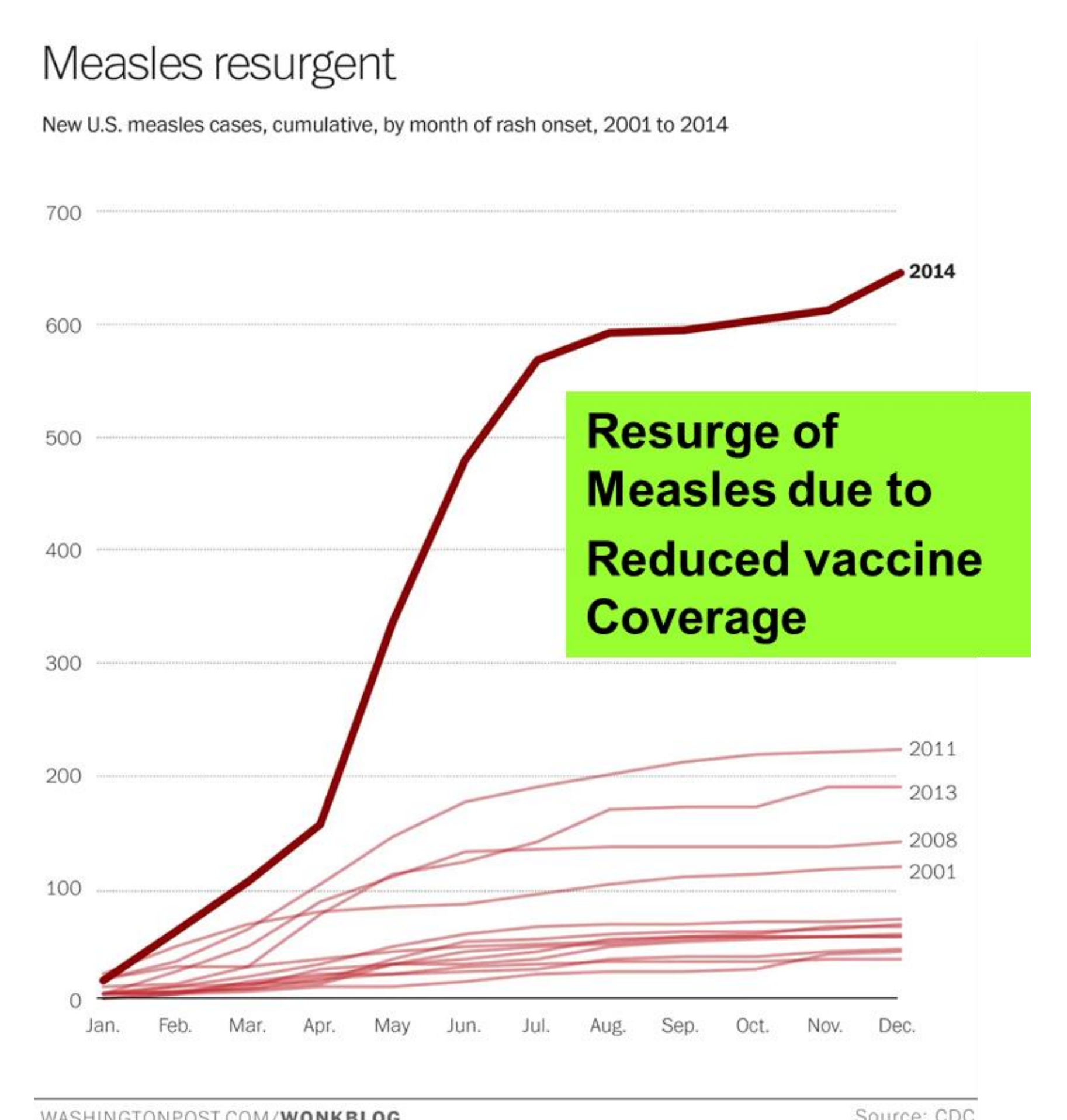

Why do we need vaccines? - Vaccines reduce disease burden in communities, save lives and money

Vaccines protect individuals against infection and prevent symptomatic illness

To date, 17 infectious diseases are prevented by vaccine administration

- Vaccine coverage impact public health
Results

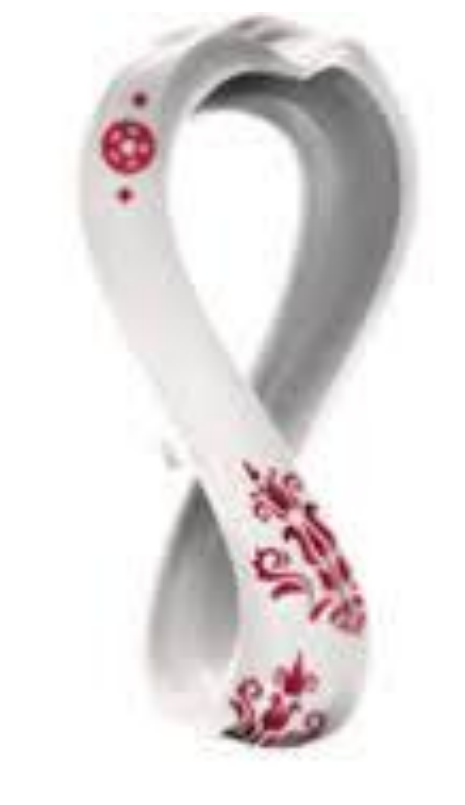

FIFA WORLD CUP Qat_ar2@22
Qatar is preparing for the upcoming FIFA World Cup 2022 therefore maintaining high vaccine coverage is critical in preventing infectious diseases spreading during such mass gathering.
GULF $\odot$ TIMES Q Q Datar tops region in providing coverage of vaccines Qatar Vaccine Coverage is $95 \%$ which is one of the highest in the world

- Seventeen infectious diseases are currently vaccine-preventable.

- The cost-effectiveness of vaccine is documented as it is estimated for each dollar spent on vaccines, 10 dollars are saved in disease treatment $(1,3)$.

- A drop in vaccine coverage rates to under $90 \%$ lead to the resurgence of measles (2).

- Vaccine coverage rates in Qatar is currently at $95 \%$ which is one of the highest in the world.

- Qatar must maintain this high coverage rate to prevent any measles outbreaks during mass gatherings.

- The planned World Cup event will take place from November 21 till December 18 2022, which is the peak for seasonal influenza.

- FIFA2022 mass gathering event, Qatar should encourage residents and visitors to be vaccinated not just against measles and seasonal influenza, but also hepatitis and meningitis.

\section{Qatar National Immunization Program}

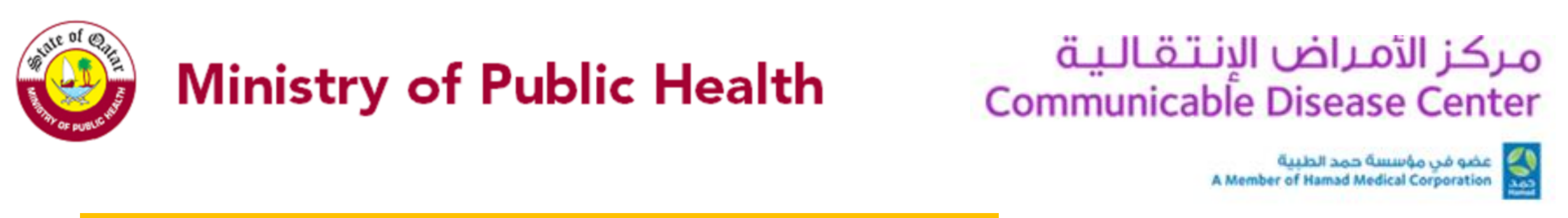

\section{Qatar Immunization Schedule}

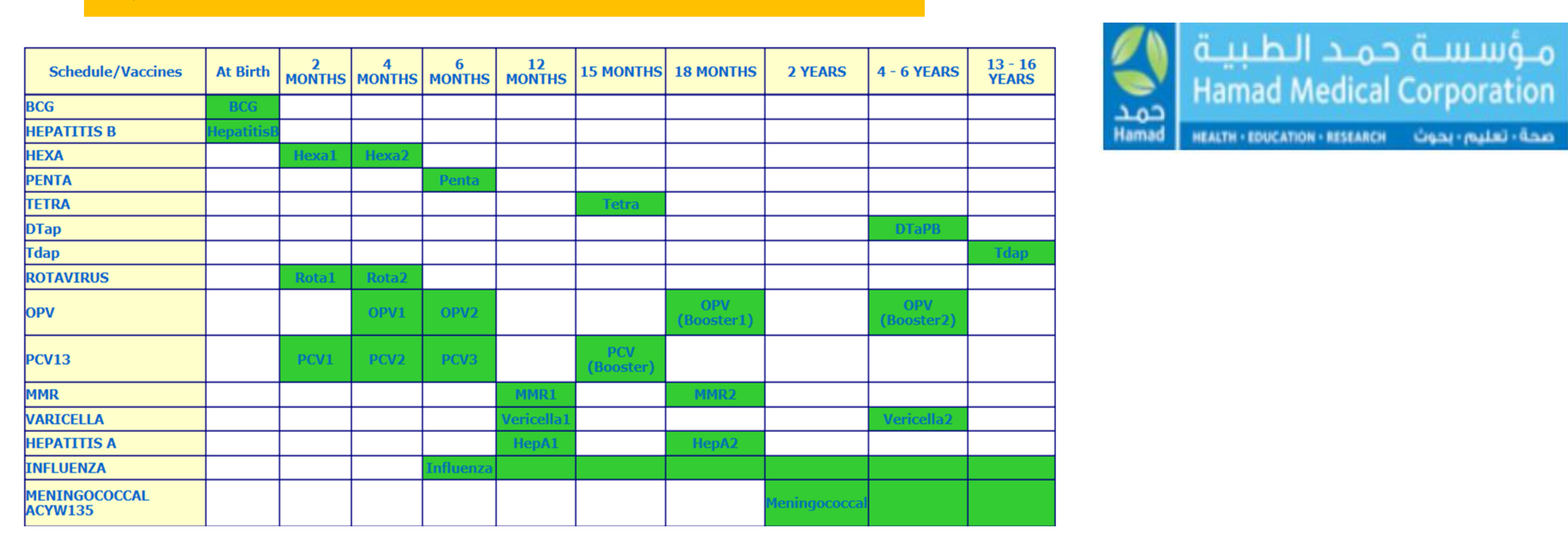

Conclusion: Maintaining 95\% vaccine coverage rate is critical for preventing the resurgence of vaccine-preventable infectious diseases during the World Cup mass gathering in Qatar.

\section{Methods}

\section{References}

1- Doherty M, Buchy $P$, Standaert B, Giaquinto $C$, Prado- Cohrs $D$. Vaccine impact: benefits for human health. Vaccine. , 34 (52), 6707-6714. 2016 Dec 20

2- Bester JC. Measles and measles vaccination: A review. JAMA Pediatr. , 170 (12), 1209 1215,2016

3- Hardt K, Bonanni P, King S, Santos Jl, El-Hodhod M, Zimet GD, Preiss S. Vaccine strategies: Optimizing outcomes. Vaccine, 34 (52), 6691-6699 2016
Methods: Literature search for vaccine coverage rates, resurgence of vaccine preventable infectious diseases and risks of mass gatherings. 